\title{
Dispersion Behavior of Gold-Nanocoated Dielectric Optical Fibers
}

\author{
P. K. Choudhury \\ Institute of Microengineering and Nanoelectronics (IMEN), Universiti Kebangsaan Malaysia, \\ Bangi, 43600 Kuala Lumpur, Malaysia \\ Correspondence should be addressed to P. K. Choudhury, pankaj@ukm.my
}

Received 21 May 2012; Revised 20 July 2012; Accepted 23 July 2012

Academic Editor: Mathrubutham Rajagopalan

Copyright ( $\odot 2012$ P. K. Choudhury. This is an open access article distributed under the Creative Commons Attribution License, which permits unrestricted use, distribution, and reproduction in any medium, provided the original work is properly cited.

Using a fairly rigorous approach, gold-nanocoated dielectric optical fibers are treated to evaluate the propagation characteristics corresponding to the transverse electric (TE) and hybrid EH modes, as supported by the guides with small and large dimensions. Situations with varying nanocoating thickness are considered for two different operating wavelengths. The results demonstrate a profound effect of the gold nanocoating on the propagation-related features-the number of existing modes decreases with increasing nanolayer thickness. Furthermore, the gold nanolayer also has effects on the modal propagation constants, which become more prominent with the increase in the order of modes. It has been found that, in fiber with smaller dimension, the $\mathrm{EH}_{11}$ mode hardly exhibits any effect due to the variation in existing nanolayer coating corresponding to $850 \mathrm{~nm}$ wavelength. Almost similar situations are observed for the $\mathrm{TE}_{01}$ mode in large core fiber operating at $1550 \mathrm{~nm}$ wavelength.

\section{Introduction}

Substantial amount of research on optical waveguides with varieties of new forms of cross-sectional geometries and/or composite materials appeared in the literature [1-10]. The electromagnetic (EM) behavior of various forms of optical fibers with different refractive index (RI) profiles and/or number of claddings have been paid much attention [1116]. However, the reported investigations mostly implement oversimplified assumptions, because a strict analytical approach, owing to the presence of multiple fibre clads, becomes forbiddingly difficult to tackle with.

Fiber optic sensors are nowadays of much demand [1720] owing to their ease of operation and environmentfriendly nature. In this context, the gold-nanocoated fibers are of special mention as these can be used with enormous benefit in chemical sensing [21-23], because the optical sensitivity of the system can be enhanced on demand by controlling the nanolayer thickness. Further, such goldnanocoated fibers remain much useful for the detection of weak chemical reactions without sacrificing the measurement sensitivity.
The knowledge of modal behavior of gold-nanocoated optical fibers has been the vital issue to secure their usefulness in sensing, particularly in medical diagnostics. This essentially needs a thorough investigation of these fibers in terms of the sustained modes and their propagation constants. Usually, in metal-nanocoated optical waveguides, the phenomenon of surface plasmon resonance (SPR) is commonly studied, and the features of modal propagation inside the guide are not given much attention. Keeping this in mind, the aim of the present communication is pivoted to the analytical treatment of gold-nanocoated optical fibers with the emphasis on the modal behavior of the guide, which forms the basics of any waveguiding medium. The structure of fiber is essentially a multilayered dielectric one coated with a metallic nanolayer. A considerable amount of refractive index (RI) difference between the different fiber regions is taken into account in order to validate the treatment with greater accuracy under the condition of minimum power leak-off. As such, the use of Maxwell's field equations becomes necessary, which makes the study rigorous, and the results are obtained with greater accuracy. 


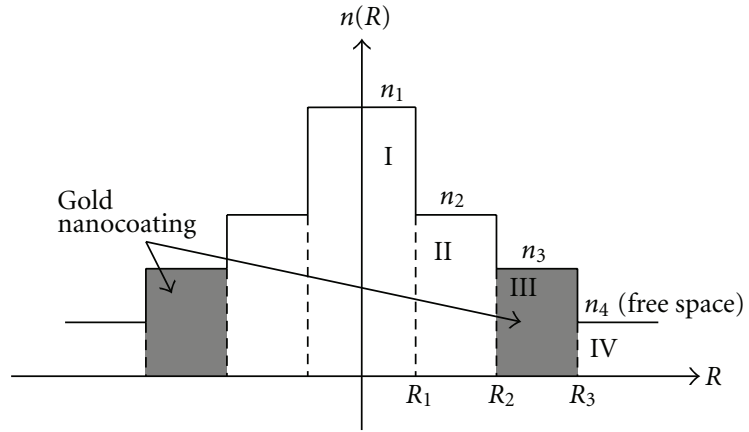

FIGURE 1: The RI profile of gold-nanocoated fiber.

The analytical treatment requires the estimation of fields in the different fiber regions, which essentially gets features due to the light-matter interaction in the guiding structure. Strictly speaking, there must be vanishing fields in the metallic region. However, as the thickness of the outermost gold-coated fiber region is of the order of nanoscale level, there must be some amount of field present in that particular region. This situation can be tackled by making use of Hankel function as one of the essential parts to represent fields in this region. Thus, a linear combination of the modified Bessel function of the second kind and Hankel function [24] is used to represent the field in the gold-nanocoated region. The present paper is aimed to investigate the effect of the gold-nanolayer thickness on the modal behavior of the guide, which ultimately provides the dispersion characteristics of the structure. In this stream, a rigorous analytical treatment is presented for gold-nanocoated fibers of small and large dimensions under the aforesaid assumptions in respect of fields, and the effect of the gold-nanolayer is visualized through the computations of the normalized propagation constants of the modes sustained in the nanocoated fiber. We consider two different operating wavelengths and investigate the effect on the propagation behavior for some of the loworder transverse electric (TE) and hybrid EH modes in the guide due to variation of the thickness of gold nanocoating.

The scope of the present paper is limited to the propagation behavior of the guide in terms of modal constraints. As such, the paper emphasizes on the dispersion characteristics of the guide in respect of the variations of the fiber dimensions and reports the observations in respect of the modal field behavior in terms of propagation cnstants. Apart from the dispersion behavior, further work in the direction is in progress and will be taken up in future communications.

\section{Analytical Treatment}

Figure 1 illustrates the RI profile of a four-layer dielectric optical fiber of which the third layer is assumed to be loaded with gold nanocoating, with $z$-axis being the optical axis. The outermost infinitely extended region is the free space (with the RI $n_{4}=1$ ). The other three layers have the RI values as $n_{1}$, $n_{2}$, and $n_{3}$ with $n_{1}>n_{2}>n_{3}$, and the interfaces at the radial distances $R=R_{1}, R=R_{2}$, and $R=R_{3}$ with $R_{1}<R_{2}<R_{3}$; the Au-nanolayer region is the one with the radial distance
$R_{3}-R_{2}(=\delta)$. We treat the problem considering the wave equation obtained under cylindrical fiber geometry [25], and matching the solutions at the layer interfaces.

Now, corresponding to the case of a gold-nanocoated fiber, the fields in the different fiber regions may be considered as follows.

Bessel function $J_{\nu}(\bullet)$ of the first kind would represent the field in the fiber core region, whereas the decaying character of the field (as one moves away from the optical axis) can be represented by the modified Bessel function $K_{\nu}(\bullet)$ of the second kind. In the region next to the fiber core, the fields should be represented by a linear combination of $J_{\nu}(\bullet)$ and $Y_{\nu}(\bullet)$, where the latter one denotes Bessel function of the second kind. Finally, a linear combination of $K_{\nu}(\bullet)$ and $H_{\nu}(\bullet)$ will represent the field in the region just before the outermost clad; here $H_{v}^{(1)}(\bullet)$ stands for Hankel function. Strictly speaking, the fields must be vanishing in the metallic region. However, the existence of some fields may always be expected in nanocoated domains, which stimulates one to implement Hankel function to partially represent the field in the gold-nanocoated region of the fiber. In all the aforesaid functions, $v$ is the mode designating parameter representing the azimuthal periodicity, and it can take only discrete values.

Following the field representations as described above, after a few mathematical steps, the use of the longitudinal components of the electric/magnetic fields will yield the tangential field components in the different regions of the guide, as expressed here.

Region I: Core $\left(0 \leq R \leq R_{1}\right)$ :

$$
\begin{aligned}
& E_{R 1}=\left\{-A_{1} \frac{j \beta}{q^{2}} Q_{1} J_{\nu}^{\prime}\left(Q_{1} R\right)+A_{2} \frac{\nu \omega \mu}{q^{2} R} J_{\nu}\left(Q_{1} R\right)\right\} e^{j \nu \phi}, \\
& H_{R 1}=-\left\{A_{1} \frac{\nu \omega \varepsilon}{q^{2} R} J_{\nu}\left(Q_{1} R\right)+A_{2} \frac{j \beta}{q^{2}} Q_{1} J_{\nu}^{\prime}\left(Q_{1} R\right)\right\} e^{j \nu \phi}, \\
& E_{\phi 1}=\left\{A_{1} \frac{\beta \nu}{q^{2} R} J_{\nu}\left(Q_{1} R\right)+A_{2} \frac{j \omega \mu}{q^{2}} Q_{1} J_{\nu}^{\prime}\left(Q_{1} R\right)\right\} e^{j \nu \phi}, \\
& H_{\phi 1}=\left\{-A_{1} \frac{j \omega \varepsilon}{q^{2}} Q_{1} J_{\nu}^{\prime}\left(Q_{1} R\right)+A_{2} \frac{\beta \nu}{q^{2} R} J_{\nu}\left(Q_{1} R\right)\right\} e^{j \nu \phi} .
\end{aligned}
$$

Region II: Inner cladding $\left(R_{1} \leq R \leq R_{2}\right)$

$$
\begin{aligned}
E_{R 2}=\{ & -A_{3} \frac{j \beta}{q^{2}} Q_{2} J_{\nu}^{\prime}\left(Q_{2} R\right)-A_{4} \frac{j \beta}{q^{2}} Q_{2} Y_{\nu}^{\prime}\left(Q_{2} R\right) \\
& \left.+A_{5} \frac{\nu \omega \mu}{q^{2} R} J_{\nu}\left(Q_{2} R\right)+A_{6} \frac{\nu \omega \mu}{q^{2} R} Y_{\nu}\left(Q_{2} R\right)\right\} e^{j \nu \phi}, \\
H_{R 2}=- & \left\{A_{3} \frac{\omega \varepsilon \nu}{q^{2} R} J_{\nu}\left(Q_{2} R\right)+A_{4} \frac{\omega \varepsilon v}{q^{2} R} Y_{\nu}\left(Q_{2} R\right)\right. \\
& \left.+A_{5} \frac{j \beta}{q^{2}} Q_{2} J_{\nu}^{\prime}\left(Q_{2} R\right)+A_{6} \frac{j \beta}{q^{2}} Q_{2} Y_{\nu}^{\prime}\left(Q_{2} R\right)\right\} e^{j \nu \phi},
\end{aligned}
$$




$$
\begin{aligned}
E_{\phi 2}= & \left\{A_{3} \frac{\beta \nu}{q^{2} R} J_{\nu}\left(Q_{2} R\right)+A_{4} \frac{\beta \nu}{q^{2} R} Y_{\nu}\left(Q_{2} R\right)\right. \\
& \left.+A_{5} \frac{j \omega \mu}{q^{2}} Q_{2} J_{\nu}^{\prime}\left(Q_{2} R\right)+A_{6} \frac{j \omega \mu}{q^{2}} Q_{2} Y_{\nu}^{\prime}\left(Q_{2} R\right)\right\} e^{j \nu \phi}, \\
H_{\phi 2}= & -A_{3} \frac{j \omega \varepsilon}{q^{2}} Q_{2} J_{\nu}^{\prime}\left(Q_{2} R\right)-A_{4} \frac{j \omega \varepsilon}{q^{2}} Q_{2} Y_{\nu}^{\prime}\left(Q_{2} R\right) \\
& \left.+A_{5} \frac{\beta \nu}{q^{2} R} J_{\nu}\left(Q_{2} R\right)+A_{6} \frac{\beta \nu}{q^{2} R} Y_{\nu}\left(Q_{2} R\right)\right\} e^{j \nu \phi} .
\end{aligned}
$$

Region III: Gold-nanocoated layer $\left(R_{2} \leq R \leq R_{3}\right)$

$$
\begin{aligned}
E_{R 3}=\{ & -A_{7} \frac{j \beta}{q^{2}} Q_{3} K_{\nu}^{\prime}\left(Q_{3} R\right)-A_{8} \frac{j \beta}{q^{2}} Q_{3} H_{\nu}^{(1)^{\prime}}\left(Q_{3} R\right) \\
& \left.+A_{9} \frac{\nu \omega \mu}{q^{2} R} K_{\nu}\left(Q_{3} R\right)+A_{10} \frac{\nu \omega \mu}{q^{2} R} H_{\nu}^{(1)}\left(Q_{3} R\right)\right\} e^{j \nu \phi}, \\
H_{R 3}=- & \left\{A_{7} \frac{\nu \omega \varepsilon}{q^{2} R} K_{\nu}\left(Q_{3} R\right)+A_{8} \frac{\nu \omega \varepsilon}{q^{2} R} H_{\nu}^{(1)}\left(Q_{3} R\right)\right. \\
& \left.+A_{9} \frac{j \beta}{q^{2}} Q_{3} K_{\nu}^{\prime}\left(Q_{3} R\right)+A_{10} \frac{j \beta}{q^{2}} Q_{3} H_{\nu}^{(1)^{\prime}}\left(Q_{3} R\right)\right\} e^{j \nu \phi}, \\
E_{\phi 3}=\{ & A_{7} \frac{\beta \nu}{q^{2} R} K_{\nu}\left(Q_{3} R\right)+A_{8} \frac{\beta \nu}{q^{2} R} H_{\nu}^{(1)}\left(Q_{3} R\right) \\
& \left.+A_{9} \frac{j \omega \mu}{q^{2}} Q_{3} K_{\nu}^{\prime}\left(Q_{3} R\right)+A_{10} \frac{j \omega \mu}{q^{2}} Q_{3} H_{\nu}^{(1)^{\prime}}\left(Q_{3} R\right)\right\} e^{j \nu \phi,} \\
H_{\phi 3}=\{ & -A_{7} \frac{j \omega \varepsilon}{q^{2}} Q_{3} K_{\nu}^{\prime}\left(Q_{3} R\right)-A_{8} \frac{j \omega \varepsilon}{q^{2}} Q_{3} H_{\nu}^{(1)^{\prime}}\left(Q_{3} R\right) \\
& \left.+A_{9} \frac{\beta \nu}{q^{2} R} K_{\nu}\left(Q_{3} R\right)+A_{10} \frac{\beta \nu}{q^{2} R} H_{\nu}^{(1)}\left(Q_{3} R\right)\right\} e^{j \nu \phi} .
\end{aligned}
$$

Region IV: outer cladding $\left(R \geq R_{3}\right)$

$$
\begin{aligned}
& E_{R 4}=\left\{-A_{11} \frac{j \beta}{q^{2}} Q_{4} K_{\nu}^{\prime}\left(Q_{4} R\right)+A_{12} \frac{\nu \omega \mu}{q^{2} R} K_{\nu}\left(Q_{4} R\right)\right\} e^{j \nu \phi}, \\
& H_{R 4}=-\left\{A_{11} \frac{\nu \omega \varepsilon}{q^{2} R} K_{\nu}\left(Q_{4} R\right)+A_{12} \frac{j \beta}{q^{2}} Q_{4} K_{\nu}^{\prime}\left(Q_{4} R\right)\right\} e^{j \nu \phi}, \\
& E_{\phi 4}=\left\{A_{11} \frac{\beta \nu}{q^{2} R} K_{\nu}\left(Q_{4} R\right)+A_{12} \frac{j \omega \mu}{q^{2}} Q_{4} K_{\nu}^{\prime}\left(Q_{4} R\right)\right\} e^{j \nu \phi}, \\
& H_{\phi 4}=\left\{-A_{11} \frac{j \omega \varepsilon}{q^{2}} Q_{4} K_{\nu}^{\prime}\left(Q_{4} R\right)+A_{12} \frac{\beta \nu}{q^{2} R} K_{\nu}\left(Q_{4} R\right)\right\} e^{j \nu \phi},
\end{aligned}
$$

In (1)-(4), $A_{1}-A_{12}$ are the unknown constants to be determined by the use of electromagnetic boundary conditions, and the suffices $1,2,3$, and 4 illustrate the corresponding fiber regions. Also, the prime represents the differentiation with respect to the argument, and the parameter $q$ is determined as

$$
q^{2}=k^{2}-\beta^{2}=\omega^{2} \mu \varepsilon-\beta^{2} .
$$

Furthermore, the core/clad parameters $Q_{1}, Q_{2}, Q_{3}$ and $Q_{4}$ are defined as

$$
\begin{aligned}
& Q_{1}^{2}=k_{1}^{2}-\beta^{2}=\omega^{2} \mu \varepsilon_{1}-\beta^{2}, \\
& Q_{2}^{2}=\beta^{2}-k_{2}^{2}=\beta^{2}-\omega^{2} \mu \varepsilon_{2}, \\
& Q_{3}^{2}=\beta^{2}-k_{3}^{2}=\beta^{2}-\omega^{2} \mu \varepsilon_{3}, \\
& Q_{4}^{2}=\beta^{2}-k_{4}^{2}=\beta^{2}-\omega^{2} \mu \varepsilon_{4} .
\end{aligned}
$$

In Equations (6), $\varepsilon_{1}, \varepsilon_{2}, \varepsilon_{3}$, and $\varepsilon_{4}$ are the dielectric constants, $\mu$ is the permeability of the medium, and $\beta$ represents the longitudinal component of the propagation constant.

Implementing the continuity condition of fields, the tangential components of the electric field $E$ and the magnetic field $H$ will exhibit smooth match at the layer boundaries represented by the parametric coordinates $R=$ $R_{1}, R=R_{2}$, and $R=R_{3}$. This yields twelve equations altogether, the consistency of which will be determined by the vanishing $(12 \times 12)$ determinant $(\Delta)$ formed by the coefficients of those equations. This indicates

$$
\Delta=0,
$$

the solutions to which will provide the modal propagation constants of the guide under consideration. Since $\Delta$ is complex, situations of the simultaneously vanishing real and imaginary parts of $\Delta$ will determine the allowed values of the propagation constants sustained by the gold-nanocoated fiber.

\section{Results and Discussion}

Equation (7) represents the dispersion relation corresponding to the gold-nanocoated fiber, as deduced by using Maxwell's field equations. The analysis becomes much rigorous to identify the sustained TE and $\mathrm{EH}$ modes. The numerical computations implement two different values of the operating wavelength $\lambda$ as $850 \mathrm{~nm}$ and $1550 \mathrm{~nm}$ - the two wavelength regions where optical waveguides are usually operated. We consider the RI values $n_{1}$ and $n_{2}$ as 1.4488 and 1.444, respectively. Also, the gold-nanocoated layer has the RI as $n_{3}=1.42$. In order to attain a comparative study, fibers with small and large dimensions are taken into consideration. In this context, for smaller dimensioned fiber, we assume parametric boundaries $R_{1}$ and $R_{2}$ as $4.1 \mu \mathrm{m}$ and $62.5 \mu \mathrm{m}$, respectively, and the outermost layer is considered to be infinitely extended. As to the fiber with large dimension, we chose the parameters as $R_{1}=50 \mu \mathrm{m}, R_{2}=12 \mu \mathrm{m}$ along with the infinite extension of the outermost layer. In both the cases, we perform investigations for varying $R_{3}$ the thickness of gold-nanolayer. We further consider that the dielectric regions of the fiber are homogeneous and isotropic along with their nature to be nonmagnetic, that is, $\mu_{1}=\mu_{2}=$ $\mu_{3}=\mu_{4} \approx \mu_{0}$, the free-space permeability. 


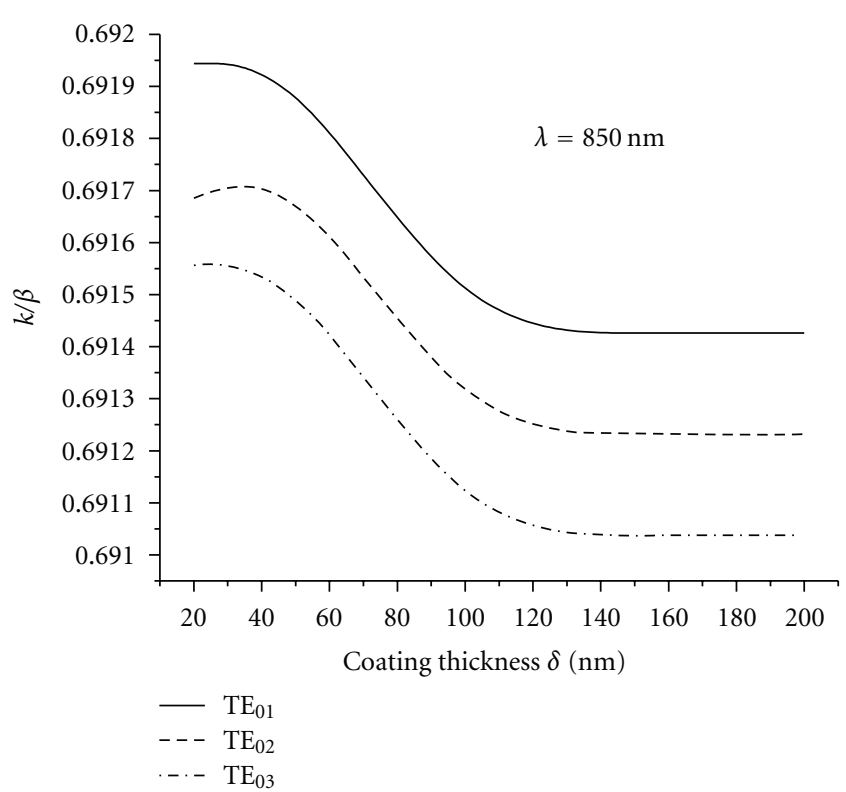

FIGURE 2: Variation of the normalized propagation constant $k / \beta$ of TE modes with the nanocoating thickness (the case of fiber with smaller dimension operating at $850 \mathrm{~nm}$ ).

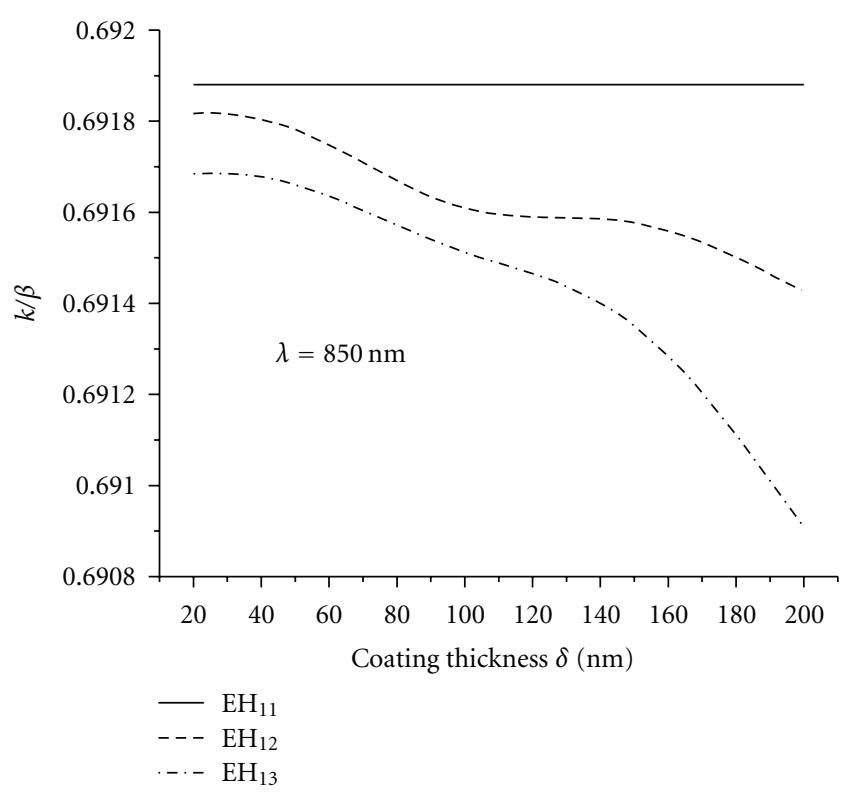

FIGURE 3: Variation of the normalized propagation constant $k / \beta$ of $\mathrm{EH}$ modes with the nanocoating thickness (the case of fiber with smaller dimension operating at $850 \mathrm{~nm}$ ).

Figures 2 and 3, respectively, illustrate the plots (corresponding to the case of small core gold-nanocoated fiber operating at $850 \mathrm{~nm}$ wavelength) of the normalized propagation constant $k / \beta$ against the nanocoating thickness $\delta$. Within the context, it is to be noted that the values of the propagation constant $\beta$ are limited by the condition of sustained guidance; that is, $n_{1} k \geq \beta \geq n_{2} k$, with $k$ being the free-space propagation constant.
In order to observe the effect of the presence of gold nanolayer on the number of sustained modes, we performed computations for several values of $\delta$, and from the study of the eigenvalue equation (i.e., (7)), we find that the number of sustained modes decreases with the increase in nanocoating thickness. This essentially reflects the feature that the increase in gold-nanolayer thickness makes the fiber to attain the property of mode suppression as the fiber gets loaded with a metallic layer in this case.

In Figure 2, we demonstrate the variation of the normalized propagation constants corresponding to the first three TE modes, namely, $\mathrm{TE}_{01}, \mathrm{TE}_{02}$, and $\mathrm{TE}_{03}$ excited at $850 \mathrm{~nm}$ wavelength. We notice that the value of $k / \beta$ initially decreases with the increase in $\delta$. This essentially indicates that, for all the three types of modes, the values of $k / \beta$ are relatively large corresponding to the situation when the gold-nanolayer thickness is small. In other words, the $\beta$ values are larger for smaller coating thickness, and the $\mathrm{TE}_{01}$ mode attains the lowest value of $\beta$ (or the highest value of $k / \beta$ ). This is very much obvious looking at the conceptual understanding of wave propagation in bounded mediums. Figure 2 also depicts that the propagation constants of TE modes saturate after the coating thickness is increased to $120 \mathrm{~nm}$ onwards. The thickness of gold nanocoating can be conceptualized through correlating it with the size of gold nanoparticles. The nanocoating thickness must have the impact on the size of grown nanoparticles-the thicker the coating is, the larger the nanoparticle size will be, affecting thereby the confinement of modes in the gold nanolayer. Figure 2 illustrates that the propagation of TE modes becomes saturated with the increase in nanocoating thickness.

Figure 3 corresponds to the situation when the $\mathrm{EH}$ modes are excited into the fiber. We observe that the propagation constants of the $\mathrm{EH}_{11}$ mode remain almost unaffected due to the variation in nanocoating thickness, which might be attributed to the specific field patterns the mode attains in the guide. However, the next higher order $\mathrm{EH}_{12}$ and $\mathrm{EH}_{13}$ modes exhibit marginal decrease in normalized propagation constant (or the increase in propagation constant) with increasing nanocoating thickness. Furthermore, the excitation of $\mathrm{EH}$ modes does not indicate any kind of saturation in the values of propagation constants with increasing $\delta$, which is in contrast to the situation of TE mode excitation, wherein $\mathrm{TE}_{01}, \mathrm{TE}_{02}$, and $\mathrm{TE}_{03}$ modes show a kind of saturation in the values of $k / \beta$ with the increase in coating thickness.

Increasing the operating wavelength (to $1550 \mathrm{~nm}$ ) results into changes in the propagation constants of the sustained TE and EH modes, as depicted in Figures 4 and 5, respectively. In both the cases the first-order modes exhibit the maximum value of $k / \beta$, which is as usual. We observe that the $\mathrm{TE}_{01}$ mode initially shows almost unchanged propagation constant values with increasing coating thickness until it reaches a value close to $120 \mathrm{~nm}$ (Figure 4). Further increase in $\delta$ results into a decrease in the values of $k / \beta$. This demonstrates partial effect of the gold-nanoparticle size on the propagation of modes for which both the field pattern as well as the nanoparticle thickness are responsible. $\mathrm{EH}$ modes also exhibit trends of initial decrease in the values of 


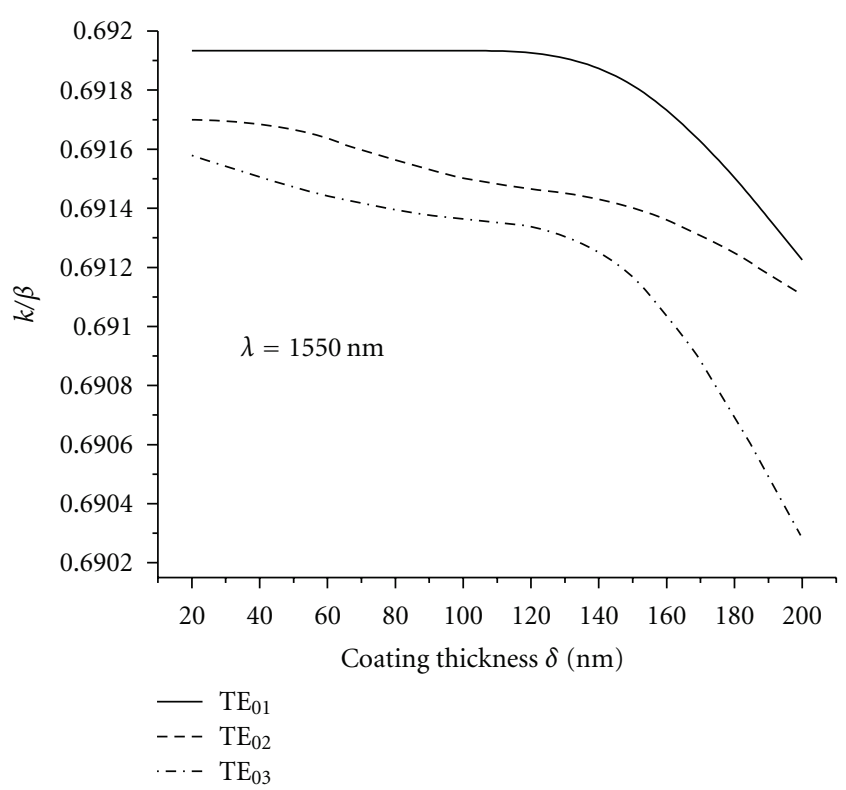

FIgURE 4: Variation of the normalized propagation constant $k / \beta$ of TE modes with the nanocoating thickness (the case of fiber with smaller dimension operating at $1550 \mathrm{~nm}$ ).

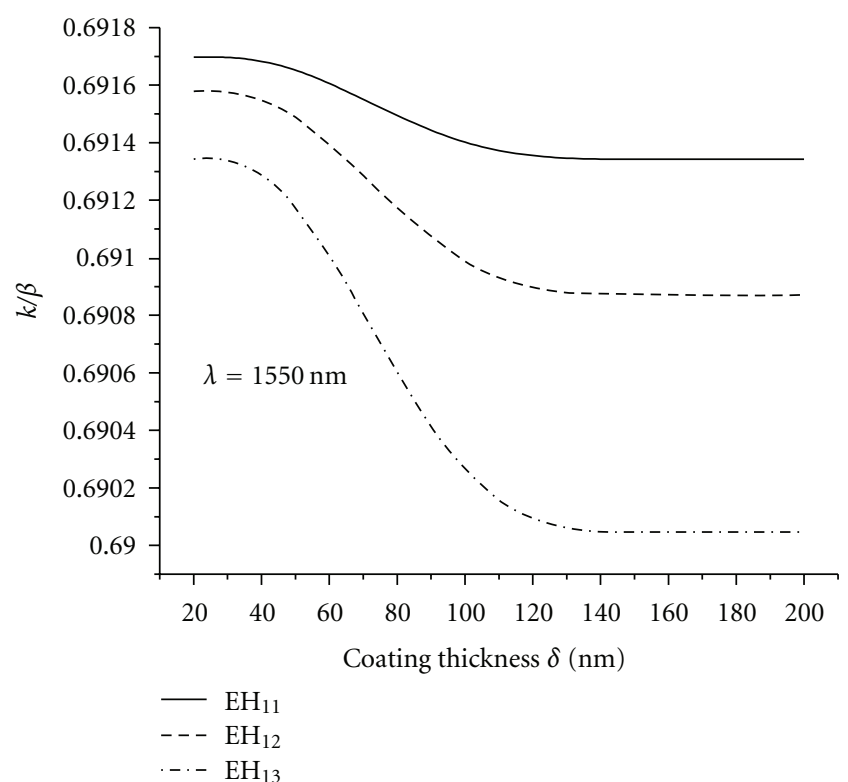

FIGURE 5: Variation of the normalized propagation constant $k / \beta$ of $\mathrm{EH}$ modes with the nanocoating thickness (the case of fiber with smaller dimension operating at $1550 \mathrm{~nm}$ ).

$k / \beta$ with increasing coating thickness and become ultimately saturated. In all the cases, we observe that the normalized propagation constant decreases with the increase in the order of mode.

The aforesaid results correspond to the situation when the gold-nanocoated fiber core size is small. However, studies can also be performed corresponding to the situation when the fiber core attains a relatively large core so that

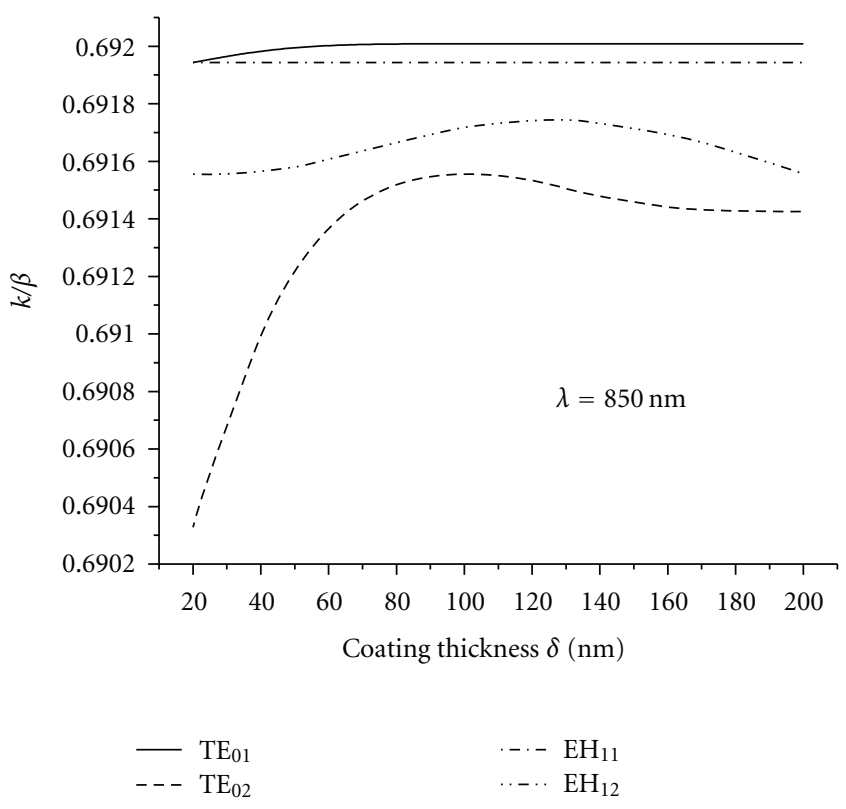

FIGURE 6: Variation of the normalized propagation constant $k / \beta$ of $\mathrm{TE}$ and $\mathrm{EH}$ modes with the nanocoating thickness (the case of fiber with larger dimension operating at $850 \mathrm{~nm}$ ).

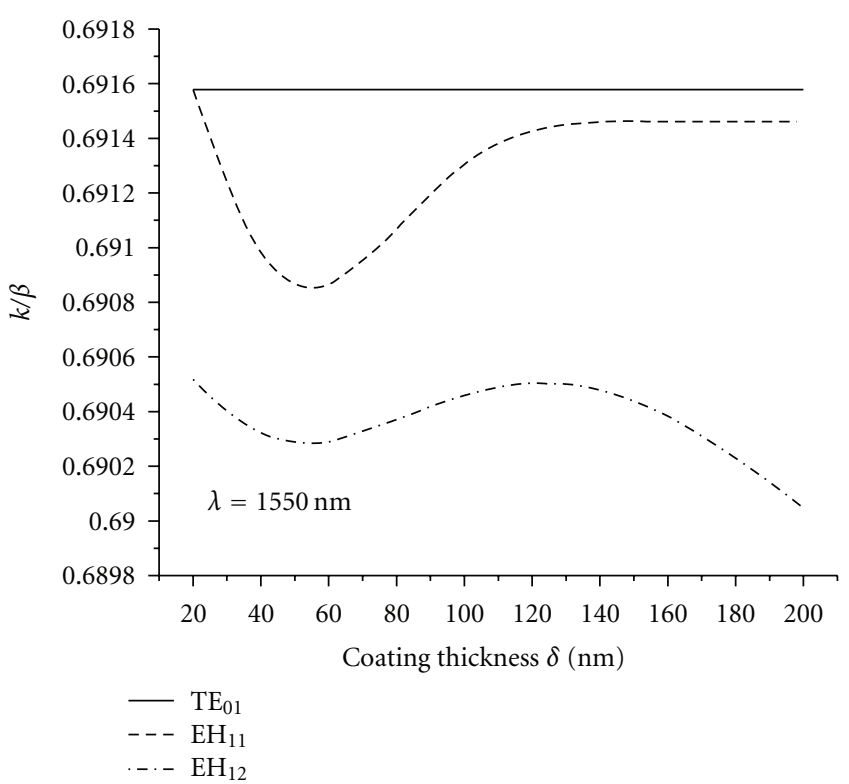

FIGURE 7: Variation of the normalized propagation constant $k / \beta$ of $\mathrm{TE}$ and $\mathrm{EH}$ modes with the nanocoating thickness (the case of fiber with larger dimension operating at $1550 \mathrm{~nm}$ ).

the field functions can be expressed in terms of their asymptotic forms under the limit of large arguments. We made computations considering such a case for operating wavelengths as $850 \mathrm{~nm}$ and $1550 \mathrm{~nm}$, and the results are illustrated in Figures 6 and 7, respectively, in the form of $k / \beta-\delta$ plots.

Interestingly, under the asymptotic limit of fields, we notice that there exist only the $\mathrm{TE}_{01}$ and $\mathrm{TE}_{02}$ modes in 
the guide (Figure 6) with the $\mathrm{TE}_{01}$ mode having marginal variation in the value of $k / \beta$ with increasing gold nanocoating thickness. The $\mathrm{TE}_{02}$ mode, however, shows larger initial increase in its normalized propagation constant, which becomes almost saturated after the coating thickness is $100 \mathrm{~nm}$ and above. We also observe from Figure 6 that the $\mathrm{EH}_{11}$ mode has hardly any dependence on the thickness value in terms of its propagation constants, and the $\mathrm{EH}_{12}$ mode exhibits a minor dependence only in similar terms. The numerical results do not reflect the existence of further higher-order modes.

Operation of large core gold-nanocoated fiber at $1550 \mathrm{~nm}$ yields further interesting results in term of the existence of modes. We find that the $\mathrm{TE}_{01}$ is the only mode that falls under the TE category, and no other mode has been observed to belong in this category. Moreover, the propagation constants of the $\mathrm{TE}_{01}$ mode remain unaffected due to the variation in the nanocoating thickness. In the $\mathrm{EH}$ mode group, as in the case of $850 \mathrm{~nm}$ operating wavelength, only the $\mathrm{EH}_{11}$ and $\mathrm{EH}_{12}$ modes are confined into the guide, and higher order modes are non existing. The trend of variation of the values of $k / \beta$ in this case has some sort of similarity with the situation when the fiber is excited at $850 \mathrm{~nm}$ operating wavelength.

The propagation of TE and EH modes in the fiber under consideration emphasizes the fact that the goldnanolayer essentially has its effect on the modal propagation constants, which become more effective when the nanolayer thickness is relatively small. For larger values of coating thickness, the propagation constants exhibit marginal dependence on the coating thickness as the larger nanoparticle size physically has less impact on the propagating field.

\section{Conclusion}

The foregoing analytical study reports on the observation of the dispersion behavior of the guide. Investigation reveals that the gold-nanocoating on dielectric optical fibers has the effect in the form of reducing the number of modes propagating in the fiber. We studied the cases of TE and $\mathrm{EH}$ modes. Relating the thickness of the nanolayer with the nanoparticle size, the effect of nanocoating has been found to be more pronounced under its small values, whereas larger coating thickness drives the system towards the situation when the larger nanoparticles exhibit less impact on the propagating fields making thereby the modal propagation constants almost saturated. Studying the case under the asymptotic limit of large core, making thereby the related field functions to be assumed to have large arguments, it has been observed that the number of modes becomes greatly reduced. Furthermore, when the fiber is operated at $850 \mathrm{~nm}$ wavelength, the low-order TE and TM modes exhibit almost unchanged values of propagation constants with the variation in nanocoating thickness. Increasing the operating wavelength to $1550 \mathrm{~nm}$, however, makes the TE mode only to remain unaffected in terms of propagation constant values due to the changes in coating thickness.
The study essentially throws information on the dispersion characteristics of gold-nanocoated optical fiber under variations of the fiber dimensions. Further studies in line with the above discussion concepts will be taken up in future communications.

\section{Acknowledgment}

The author is thankful to an anonymous reviewer for making some comments on the text, which caused to enhance the descriptions.

\section{References}

[1] P. K. Choudhury and O. N. Singh, "Some multilayered and other unconventional lightguides," in Electromagnetic Fields in Unconventional Materials and Structures, O. N. Singh and A. Lakhtakia, Eds., chapter 8, John Wiley \& Sons, New York, NY, USA, 2000.

[2] D. Kumar, P. K. Choudhury, and F. A. Rahman, "Towards the characteristic dispersion relation for step-index hyperbolic waveguide with conducting helical winding," Progress in Electromagnetics Research, vol. 71, pp. 251-275, 2007.

[3] C. Yeh and I. F. Shimabukuro, The Essence of Dielectric Waveguides, Springer, New York, NY, USA, 2008.

[4] D. Kumar, P. K. Choudhury, and O. N. Singh, "Towards the dispersion relations for dielectric optical fibers with helical windings under slow- and fast-wave considerationsa comparative analysis," Progress in Electromagnetics Research, vol. 80, pp. 409-420, 2008.

[5] A. S. N. Amin, M. Mirhosseini, and M. Shahabadi, "Modal analysis of multilayer conical dielectric waveguides for azimuthal invariant modes," Progress in Electromagnetics Research, vol. 105, pp. 213-229, 2010.

[6] V. Kesari and J. P. Keshari, "Analysis of a circular waveguide loaded with dielectric and metal discs," Progress in Electromagnetics Research, vol. 111, pp. 253-269, 2011.

[7] P. K. Choudhury and W. K. Soon, "On the tapered optical fibers with radially anisotropic liquid crystal clad," Progress in Electromagnetics Research, vol. 115, pp. 461-475, 2011.

[8] P. K. Choudhury and P. T. S. Ping, "On the dispersion relations of tapered coreoptical fibers with liquid crystal clad," Progress in Electromagnetics Research, vol. 118, pp. 117-133, 2011.

[9] D. Zarifi, A. Abdolali, M. Soleimani, and V. Nayyeri, "Inhomogeneous planar layered chiral media: analysis of wave propagation and scattering using Taylor's series expansion," Progress in Electromagnetics Research, vol. 125, pp. 119-135, 2012.

[10] J. Dong and J. Li, "Characteristics of guided modes in uniaxial chiral circular waveguides," Progress in Electromagnetics Research, vol. 124, pp. 331-345, 2012.

[11] V. K. Chaubey, K. K. Dey, S. P. Ojha, and P. Khastgir, "Modal characteristics of a doubly clad step-index optical fibre: a general analytical approach," Canadian Journal of Physics, vol. 66, no. 9, pp. 796-802, 1988.

[12] P. K. Choudhury, P. Khastgir, S. P. Ojha, and L. K. Singh, "Weak guidance in bent and unbent four-layer planar waveguides. A comparative study of dispersion curves," Japanese Journal of Applied Physics, Part 2, vol. 31, no. 1, pp. L39-L42, 1992. 
[13] P. K. Choudhury and R. A. Lessard, "An estimation of power transmission through a doubly clad optical fiber with an annular core," Microwave and Optical Technology Letters, vol. 29, no. 6, pp. 402-405, 2001.

[14] Z. Duan, Y. Wang, X. Mao, W. X. Wang, and M. Chen, "Experimental demonstration of double-negative metamaterials partially filled in a circular waveguide," Progress in Electromagnetics Research, vol. 121, pp. 215-224, 2011.

[15] D. Chen and B. Sun, "Multi-wavelength fiber optical parametric oscillator based on a highly nonlinear fiber and a sagnac loop filter," Progress in Electromagnetics Research, vol. 106, pp. 163-176, 2010.

[16] B. Zhu, J. Stiens, V. Matvejev, and R. Vounckx, "Inexpensive and easy fabrication of multi-mode tapered dielectric circular probes at millimeter wave frequencies," Progress in Electromagnetics Research, vol. 126, pp. 237-254, 2012.

[17] P. H. Paul and G. Kychakoff, "Fiber-optic evanescent field absorption sensor," Applied Physics Letters, vol. 51, no. 1, pp. 12-14, 1987.

[18] A. Messica, A. Greenstein, and A. Katzir, "Theory of fiberoptic, evanescent-wave spectroscopy and sensors," Applied Optics, vol. 35, no. 13, pp. 2274-2284, 1996.

[19] P. K. Choudhury and T. Yoshino, "On the fiber-optic chlorine sensor with enhanced sensitivity based on the study of evanescent field absorption spectroscopy," Optik, vol. 115, no. 7, pp. 329-333, 2004.

[20] P. K. Choudhury and O. N. Singh, "An overview of optical sensors and their applications," in Frontiers in Optical Technology: Materials and Devices, chapter 9, Nova Science, New York, NY, USA, 2007.

[21] S. F. Cheng and L. K. Chau, "Colloidal gold-modified optical fiber for chemical and biochemical sensing," Analytical Chemistry, vol. 75, no. 1, pp. 16-21, 2003.

[22] A. K. Sharma, R. Jha, and B. D. Gupta, "Fiber-optic sensors based on surface plasmon resonance: a comprehensive review," in IEEE Sensors Journal, vol. 7, pp. 1118-1129, 2007.

[23] F. A. Rahman, P. K. Choudhury, D. Kumar, and Z. Yusoff, "An analytical investigation of four-layer dielectric optical fibers with Au nano-coating - a comparison with three-layer optical fibers," Progress in Electromagnetics Research, vol. 90, pp. 269286, 2009.

[24] M. Abramowitz and I. A. Stegun, Handbook of Mathematical Functions, chapter 9, Dover Publications, New York, NY, USA, 1965.

[25] G. Keiser, Optical Fiber Communications, chapter 2, McGrawHill, Singapore, 1986. 

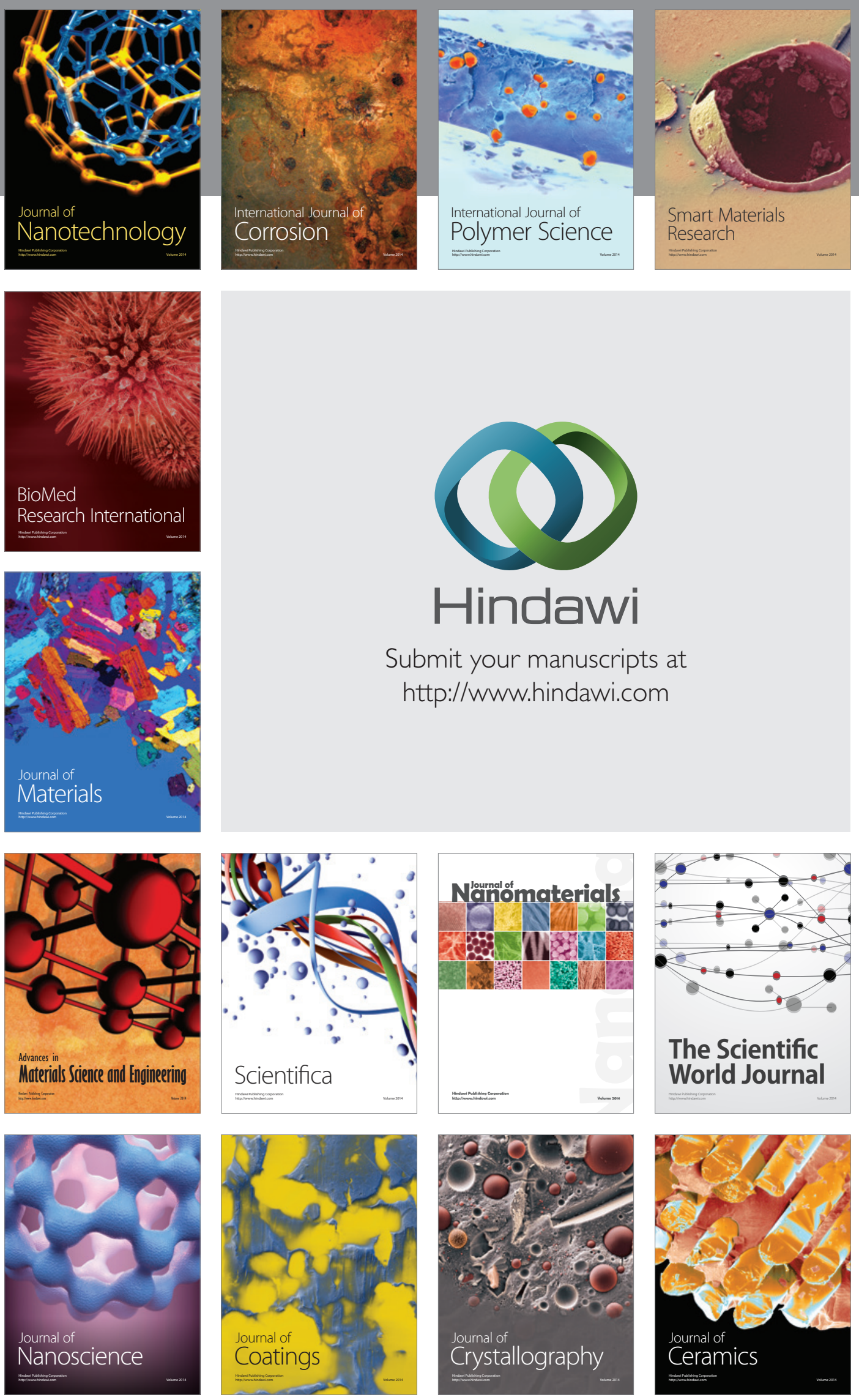

The Scientific World Journal

Submit your manuscripts at

http://www.hindawi.com

\section{World Journal}

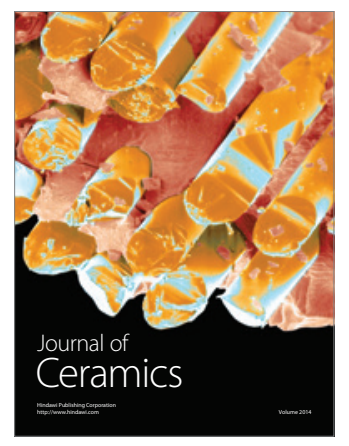

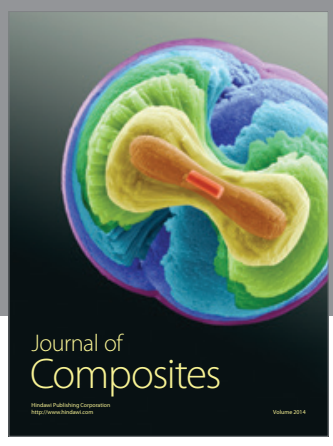
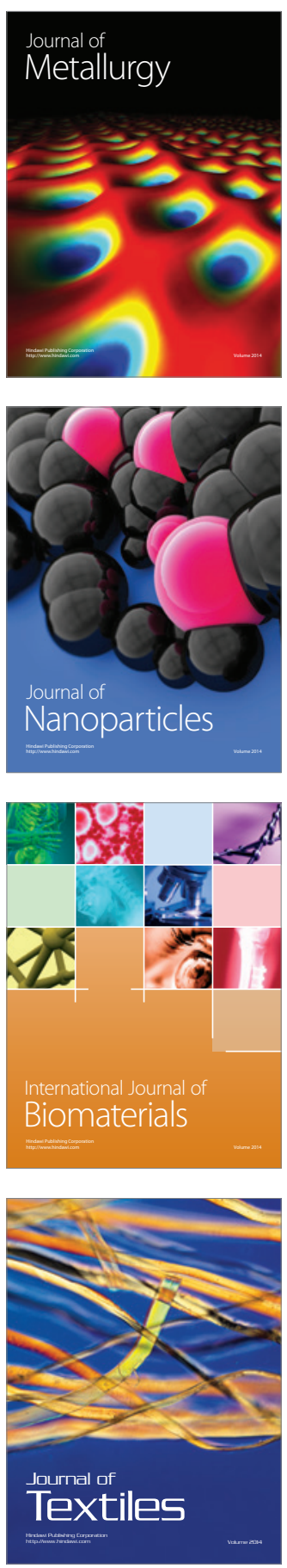